\title{
AIAA-99-3575 \\ Transitioning Active Flow Control to Applications
}

Ronald D. Joslin, Lucas G. Horta, and Fang-Jenq Chen NASA Langley Research Center Hampton, VA 
AIAA-99-3575

\title{
TRANSITIONING ACTIVE FLOW CONTROL TO APPLICATIONS
}

\author{
Ronald D. Joslin ${ }^{*}$ Lucas G. Horta ${ }^{\dagger}$, and Fang-Jenq Chen ${ }^{*}$ \\ NASA Langley Research Center ${ }^{1}$, Hampton, VA 23681
}

\begin{abstract}
Active Flow Control Programs at NASA, the U.S. Air Force, and DARPA have been initiated with the goals of obtaining revolutionary advances in aerodynamic performance and maneuvering compared to conventional approaches. These programs envision the use of actuators, sensors, and controllers on applications such as aircraft wings/tails, engine nacelles, internal ducts, nozzles, projectiles, weapons bays, and hydrodynamic vehicles. Anticipated benefits of flow control include reduced weight, part count, and operating cost and reduced fuel burn (and emissions), noise and enhanced safety if the sensors serve a dual role of flow control and health monitoring. To get from the bench-top or laboratory test to adaptive distributed control systems on realistic applications, reliable validated design tools are needed in addition to sub- and large-scale wind-tunnel and flight experiments. This paper will focus on the development of tools for active flow control applications.
\end{abstract}

\section{INTRODUCTION}

Increased attention has been devoted to the development of techniques ${ }^{1,2}$ capable of enhancing our ability to control unsteady flows in a wide variety of configurations and applications. Controlling the flow in and around these configurations can lead to greatly improved efficiency and performance. While passive devices, such as the micro vortex generator, ${ }^{3,4}$ have been incorporated into production vehicles, passive devices are primarily limited to a single function or are effective over small operational ranges. Although decades of research have been conducted using pseudo-active controls (e.g., suction laminar flow control ${ }^{5}$ ), questions of system complexity and/or cost versus benefit have prevented such controls from reaching marketable applications. With the recent introduction of novel actuator concepts, which are locally applied and require only electrical power input, the potential for vehicle control without conventional control surfaces may be within our reach while decreasing the weight and cost of a conventional vehicle. ${ }^{6}$

In recent years, a limited number of active flow control applications are being tested in the laboratory. These applications include dynamic stall control using a deformable leading edge, ${ }^{7}$

\footnotetext{
* Senior Research Scientist, Flow Modeling and Control Branch, Senior Member.

${ }^{\dagger}$ Assistant Head, Structural Dynamics Branch, Senior Member.

${ }^{1}$ Copyright (C) 1999 by the American Institute of Aeronautics and Astronautics, Inc. No copyright is asserted in the United States under Title 17, U.S. Code. The U.S. Government has a royalty-free license to exercise all rights under the copyright claimed herein for Government Purposes. All other rights are reserved by the copyright owner.
} 
separation control for takeoff and landing flight conditions using piezo devices, ${ }^{8}$ pulsed vortex generators, ${ }^{9}$ and zero-net-mass oscillations, ${ }^{10,11}$ duct-flow separation control and fore-body vortex control using zero-net-mass suction and blowing, and thrust vectoring with zero-net-mass oscillatory actuation. ${ }^{12}$ Although the continued demonstration of active flow control in the laboratory will continue for many years, other research areas such as power management and electronics for tens to hundreds of self-adaptive controller systems, the life-cycle and/or degradation of the active devices with operation, impact of the embedded devices on the structural integrity of the vehicle, and control management for local and distributed failure modes require a mature level of understanding and predictability prior to bringing the flow control from the laboratory to real applications. Finally, design tools which mimic the real systems involved in an active flow control system/application must be developed and validated with laboratory and flight databases. Our current capabilities in design tools for active flow control will be outlined for this paper. A subsequent paper will outline the status of hardware and manufacturing process issues related to flow control; issues related to the isolated actuator are covered in this paper.

\section{COMPUTATIONAL FLUID DYNAMICS}

This section will highlight some of the goals and tools that require additional computational research to enable efficient robust design tools for flow control on complex geometries. Some of these goals include "time-accurate" Reynolds Averaged Navier-Stokes (RANS) validation with boundary conditions that physically mimic the actuators, ${ }^{13}$ robust and optimal control methodologies, ${ }^{14}$ reduced order basis methodologies, ${ }^{15}$ and modeling which links structural and fluid dynamics for actuator design. For brevity, this paper will concentrate on RANS validation and boundary conditions and structuralfluid modeling for synthetic jet actuators.
For flow control applications, the NASA CFL3D (structured) and FUN2D and USM3D (unstructured) codes are being validated for timeaccuracy and then suitable actuator boundary conditions are being implemented. Numerous time-varying experimental data are available for validating time-accurate codes on simplified geometries and without actuation. Some limited steady actuation (e.g., steady blowing) experiments are also available to initially test the boundary conditions. More recently, experiments carried out at Tel-Aviv University and NASA Langley Research Center with wing and wing-like models, fore-body control, and thrust vectoring jets now permit the validation of time-accurate CFD for active flow control applications. Unsteady blowing and zero-net-mass suction and blowing actuators have been used to control the flow in flight regimes ranging from low-speed incompressible through transonic conditions and at flight Reynolds numbers.

A number of systematic studies must first be carried out to validate the codes before they are ready for flow control applications. A sample of the results to date for these studies is presented in the remaining sections using first the FUN2D code on a workstation class computer. Future papers will compare/contrast CFL3D, FUN2D, and USM3D.

The FUN2D code $^{16}$ solves the time-dependent Reynolds Averaged Navier-Stokes equations expressed as a system of conservation laws relating the rate of change of mass, momentum, and energy in a control volume to the fluxes of these quantities through the control volume. The solver is an implicit, upwind differencing algorithm with the inviscid fluxes obtained on each face of the control volume, employing Roe's fluxdifference-splitting scheme. The node-based algorithm stores the variables at the vertices of the 
mesh and the equations are solved on the control volumes surrounding each node. The viscous terms use a central-difference-type formulation evaluated with the finite-volume formulation.

Time-advancement is made with a backward-Euler scheme. At each time step, the equations are solved with a sub-iterative approach, sequentially solving for all odd numbered nodes and then all even numbered nodes. For more specific details of the FUN2D code refer to Ref. 16.

\subsection{TAU0015 Baseline Configuration}

The first study involves determining the sensitivity of the solution to potential complex geometries (e.g., actuator discontinuities) with baseline configurations (no actuators). An understanding of this sensitivity should indicate which details of the configuration must be resolved and which details can be "fared over" for more efficient solution methodologies.

For this presentation, an active flow control wing configuration, which was tested in a low-speed wind tunnel at the Tel-Aviv University (TAU), is used first to determine the sensitivity of the aerodynamic performance to discontinuities in the geometry. For this baseline study, all results shown in this section use no actuation. The experimental conditions were at a Mach number of 0.15 and a chord Reynolds numbers of 1.2 million. The SA turbulence mode ${ }^{17}$ was used for all airfoil computations.

Although not the optimal multi-grid formulation, the results presented here used a two-grid multi-grid approach for this baseline study. There was no attempt here to optimize the system for efficiency, rather a very fine grid was used to determine sensitivities of the discontinuities, while minimizing the grid-dependent influences. Future studies will have the goal of minimizing the grid requirements and optimizing the multi-grid usage. For the fine grid surrounding the TAU airfoil, the node-base scheme has 114,119 nodes, with 1891 of the nodes on the surface. The coarse grid had 51,150 nodes, with 1260 nodes on the surface. This baseline study only uses mean converged solutions; hence, angles of attack where periodic vortex shedding occurs are avoided.

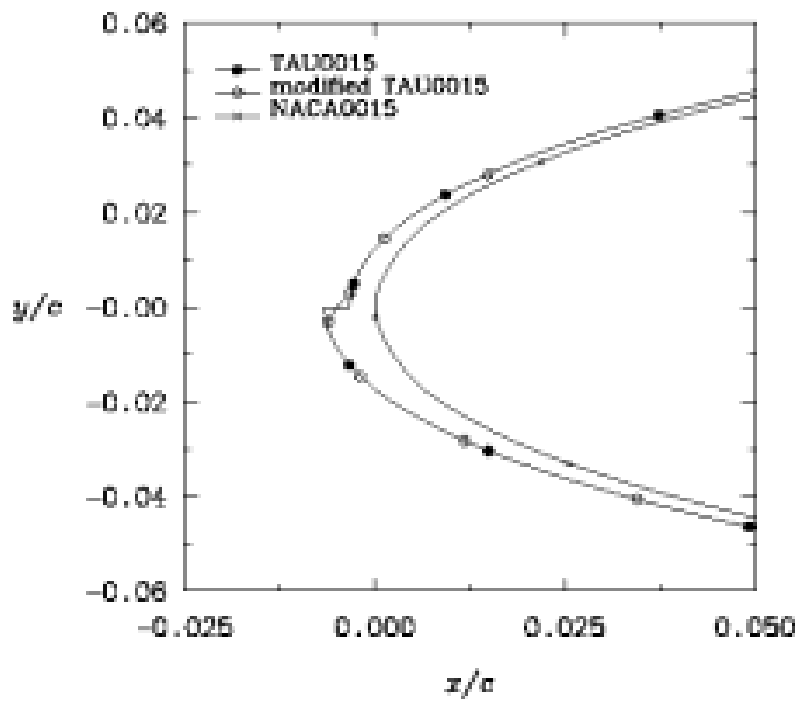

Figure 1. Leading-edge regions of NACA 0015, TAU0015, and modified TAU0015 airfoils.

Figure 1 shows a comparison of the standard NACA 0015 airfoil with the experimentally tested TAU015 airfoil and computationally tested modified TAU0015 airfoil. The modified TAU0015 airfoil enabled an efficient structured grid computation (with a single-block grid). ${ }^{18}$ The TAU0015 model has a 0.4 percent chord notch at 76.6 percent chord, which results from the flap/main element connection, and a 3-percent chord thick trailing edge. The actuator for the TAU0015 tests was located at the leading edge and leads to the discontinuity at 0 percent chord. To study the sensitivity of the leading edge and mid-chord discontinuities, FUN2D computations were made with the NACA 0015, TAU0015, and modified TAU0015 airfoils and are presented in the remainder of this section. 

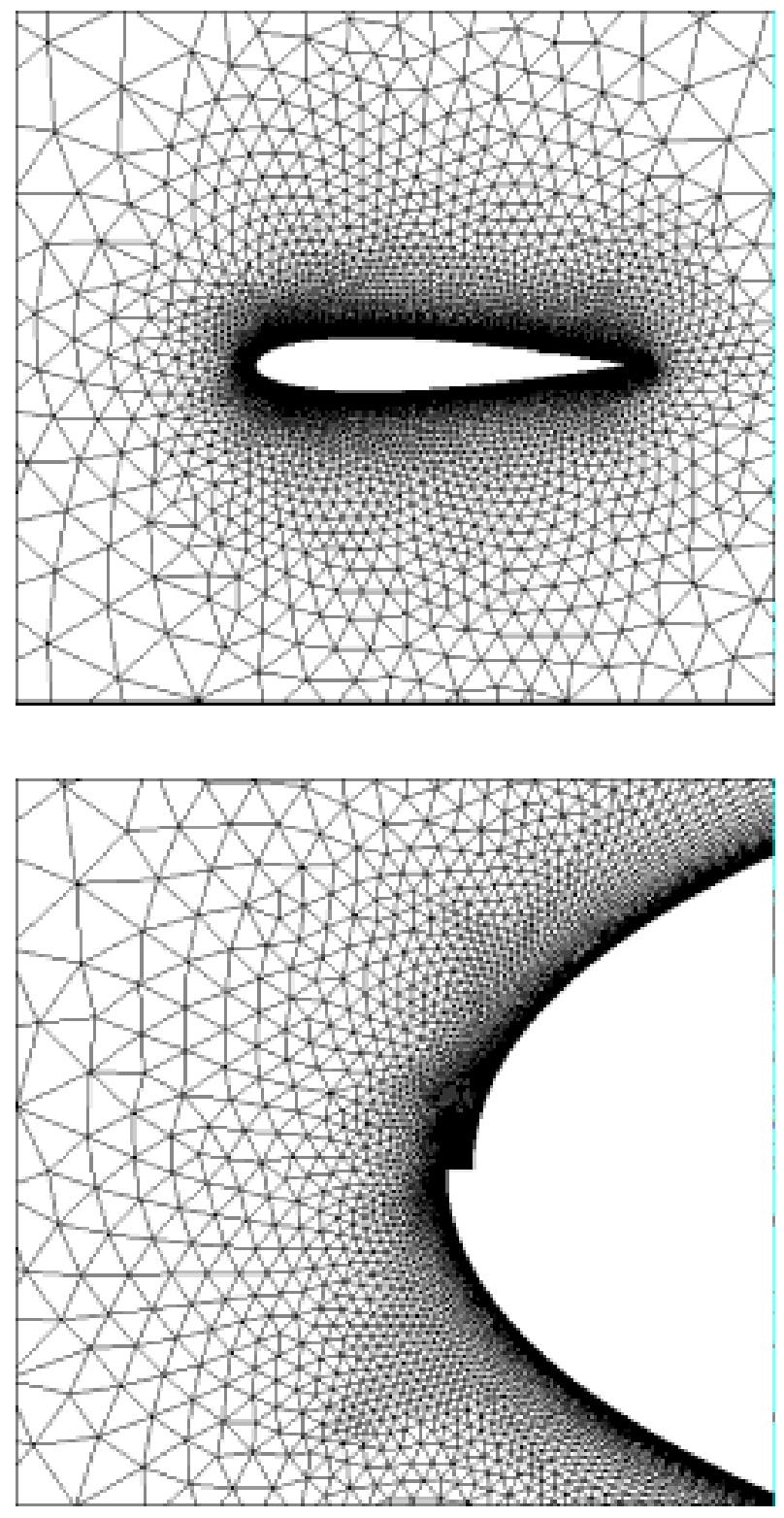

Figure 2. Unstructured coarse grid around TAU0015 airfoil and enlarged view around leading-edge discontinuity.

Figure 2 shows the coarse grid around the TAU0015 airfoil. Similar grids were generated for modified TAU0015 and NACA 0015 airfoils. The complete grids extend 20 chord lengths above, below, upstream and downstream of the airfoil.

Velocity contours and vectors around the TAU0015 airfoil indicate that the RANS computations capture what appear to be recirculation zones associated with the leading edge, trailing edge, and the 76-percent-chord notch discontinuities. These re-circulation zones physically seem plausible (albeit no quantitative comparisons are possible for this configuration).

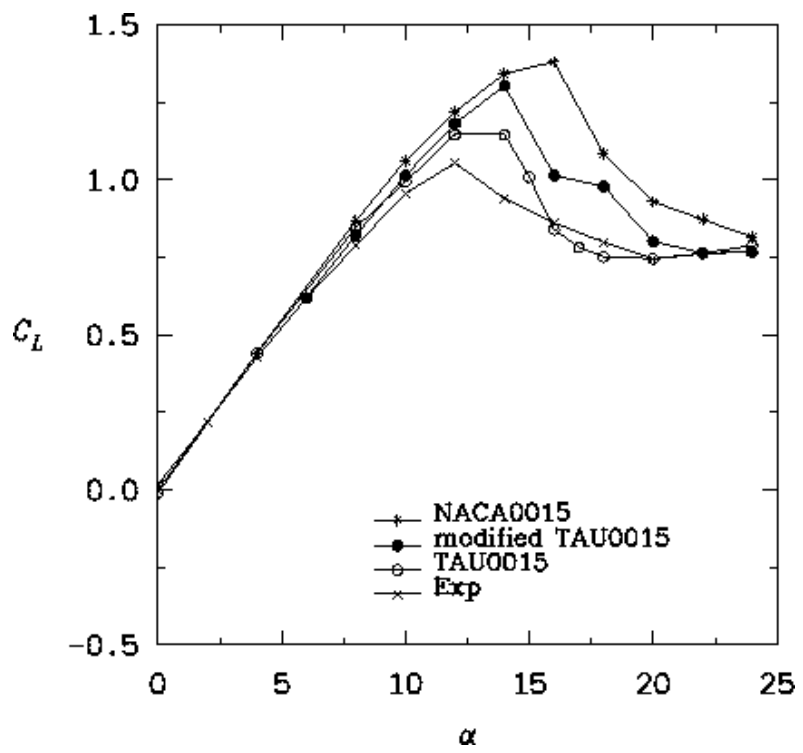

Figure 3. Computed lift coefficients $\left(C_{L}\right)$ with angle of attack $(\alpha)$ for the NACA 0015, TAU0015 and modified TAU0015 configurations compared with the TAU experiments.

Figure 3 shows the computed lift coefficient $\left(C_{L}\right)$ with variation in angle of attack for the NACA 0015, TAU0015, and modified TAU0015 airfoils compared with the experimental data. The computed NACA 0015 airfoil results are significantly different compared with the experimental data from the TAU0015 model. The computed results for the "faired-over actuator" model are remarkably close to the computed TAUmodel results, except the TAU0015 model stalls before the modified TAU0015 model and hence shows some disagreement in the highly separated region after stall. This may suggest that a more efficient structured grid approach may be used for parameter studies of slightly altered geometries involving faired-over actuators for flow control. However, the computations and comparisons must 
be repeated with actuation to truly ascertain the role of geometry and discontinuities in flow control applications. Finally, significant disagreement exists between the three computational results compared with the experimental data. A similar disagreement is evident in the Ref. 18 comparisons, even when different turbulence models were used in the computations.

Using the exact same geometry (TAU0015 model) in both computations and experiments, most of the discrepancy in the results can be explained in light of a proper interpretation of the experimental results. Namely, the bullets shown in Figure 1 for the TAU0015 model are the discrete pressure tap locations used in the experiments. Hence, the lift coefficient consists of the pressure contribution only, and the region near the leading-edge actuator is not considered in computing lift/drag. Based on this understanding of the experimental lift coefficients, the lift is re-computed using the pressure while neglecting the contribution from the leading-edge actuator discontinuity. The comparison in Figure 4 indicates a nearly exact agreement between the computational and experimental data, including the identical angles for stall. Some small disagreement remains in the separated angle-of-attack region; however, some uncertainty in the experimental values exists because of the sensitivity of the flow in this region.

This section has demonstrated that discrepancies in the computed aerodynamic properties can be attributed to local discontinuities associated with geometry variation and actuators placement. However, some of these discrepancies can be minimized by "fairing over" discontinuities to enable parameter studies with more efficient structured codes. Some additional discrepancies between computational and experimental performance can be linked to variations in the methods used to determine the aerodynamic properties.

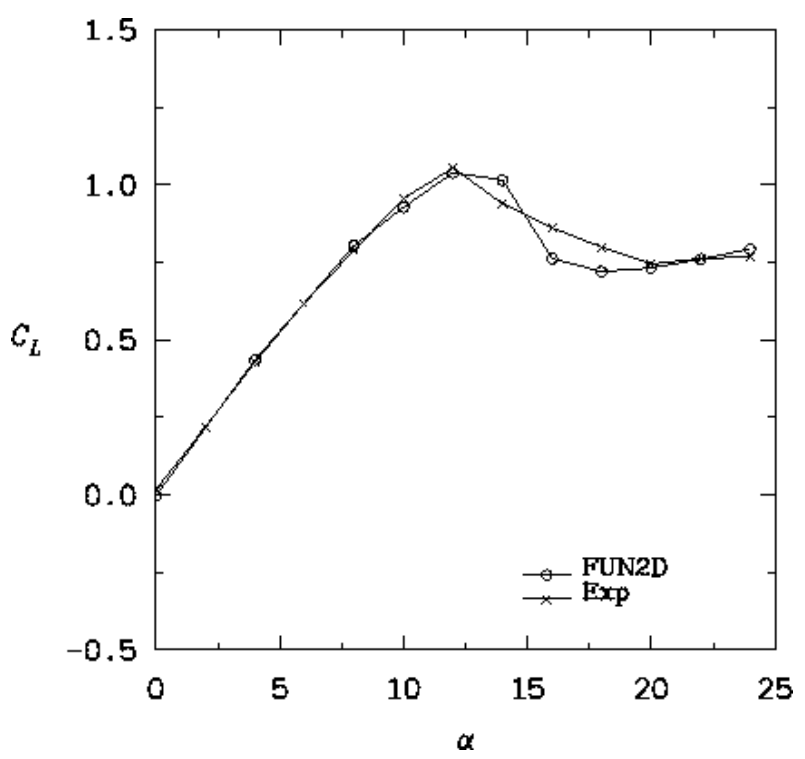

Figure 4. Computed lift coefficient $\left(\mathrm{C}_{\mathrm{L}}\right)$ with angle of attack $(\alpha)$ compared with the TAU experiments.

\subsection{Time-Accurate Validation}

The second study involves determining the requirements for time-accurate CFD. The timeaccuracy of FUN2D is validated for periodic vortex shedding from circular-cylinder, square, and NACA 0012 airfoil geometries. These test cases are used because a significant number of published experimental and computational (structured-code) databases exist for comparison with the current RANS approach. A single grid is used for the cylinder and square test cases and a three-level multi-grid approach is used for the NACA 0012 airfoil case.

The results in this paper will highlight only the cylinder result. Although the geometry is "trivial," the flow field and vortex shedding process are complex. ${ }^{19}$ Depending on the Reynolds number, the vortex shedding process changes from lowReynolds number, laminar, two-dimensional shedding to higher Reynolds number, turbulent, three-dimensional shedding. In addition, boundary layer, shear layer, and wake flows can be laminar, 
transitional, or turbulent, depending on the Reynolds number.

To begin the validation process for the RANS code, we begin with low-Reynolds number laminar shedding to enable efficient parameter studies. No turbulence model is used in this first study. Computations with Mach numbers (Ma) ranging from 0.1 to 0.3 indicate a relatively insignificant sensitivity of the results to Mach number; hence, all of the results have a Mach number of 0.15 . A Reynolds number $(\mathrm{Re})$ based on the free-stream velocity, kinematic viscosity, and a unit-length cylinder diameter of 300 is selected. The grid around the cylinder extends 20 diameters from the cylinder and has 47,607 nodes, with 800 of the nodes on the surface.

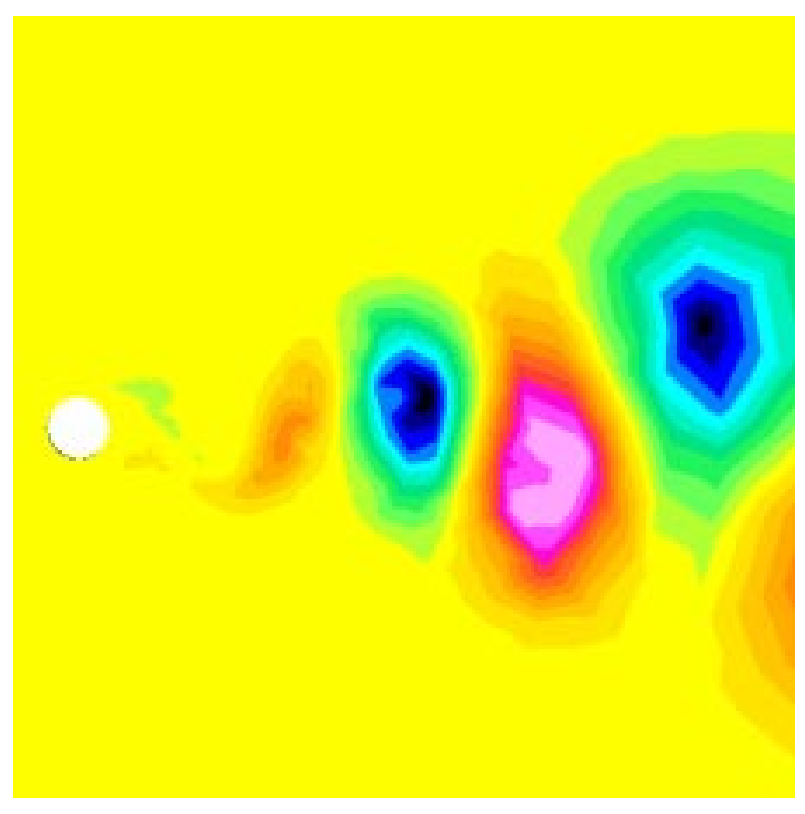

Figure 5. Computed instantaneous vorticity contours from FUN2D for $\mathrm{Re}=300, \mathrm{Ma}=0.15$, $\mathrm{dt}=0.05$.

Unlike the mean flow solutions sought in the previous complex airfoil study, the current test cases require an understanding of the sensitivity of the solution to time advancement. To accelerate the solution procedure, a large CFL time advancement was made to obtain "meanlike" flow fields. Then time-accurate timeadvancement was carried out to get timedependent (and hopefully time-accurate) flow fields. Figure 5 shows a snapshot in time of the time-accurate vorticity field.

The sensitivity of the final solution to time-step size is shown in Table 1. A representative published solution ${ }^{20}$ reproduced in our Table 2 suggests that the large time-step size $(\mathrm{dt}=0.5)$, which is approximately 65 steps per period of shedding, leads to unacceptable errors in the Strouhal number (shedding frequency) and maximum lift and mean drag coefficients. For these low Reynolds numbers, time advancement with approximately 300 steps per period of shedding yields sufficiently good resolution to permit parameter studies. A future paper will document time-accuracy for large Reynolds numbers and turbulent flows.

Table 1. Time-step variation for flow past cylinder.

\begin{tabular}{|c|l|c|c|c|}
\hline $\mathrm{Re}$ & \multicolumn{1}{|c|}{$\mathrm{dt}$} & $\mathrm{St}$ & $\mathrm{C}_{\mathrm{L}}(\max )$ & $\mathrm{C}_{\mathrm{D}}$ (ave) \\
\hline 300 & 0.5 & 0.128 & 0.685 & 1.2 \\
& 0.1 & 0.192 & 0.759 & 1.28 \\
& 0.05 & 0.197 & 0.805 & 1.31 \\
& 0.01 & 0.2026 & 0.806 & 1.32 \\
& 0.007 & 0.2032 & 0.841 & 1.33 \\
& 0.0035 & 0.2036 & 0.841 & 1.34 \\
\hline
\end{tabular}

Table 2. Published results for flow past cylinder. ${ }^{20}$

\begin{tabular}{|c|c|c|c|c|}
\hline$R e$ & $d t$ & $S t$ & $C_{L}$ & $C_{D}$ \\
\hline 300 & 0.05 & 0.205 & 0.84 & 1.32 \\
\hline
\end{tabular}

\subsection{Actuator Boundary Conditions}

For active flow control, locally (potentially oscillating) surfaces (e.g., Shape-Memory Alloy motion), local blowing, suction and/or oscillatory 
suction and blowing boundary conditions are required for design tools. Here, we will focus on fluidic actuation. Initial research to develop adequate (yet simple) boundary conditions ${ }^{13}$ to mimic the synthetic jet actuator ${ }^{21}$-zero-net-mass suction and blowing actuator that generates a turbulent-like jet-proved to reproduce the actuator-induced turbulent flow quantities measured in the experiments. Replacing the noslip condition only on the actuator region, a simple boundary condition can be introduced to support steady suction/blowing, an oscillatory (zero-netmass) component, or a combination of steady and oscillatory actuation. This boundary condition is given by

$$
v(x, t)=A_{0} f_{0}(x)+A_{1} f_{1}(x) \sin (\omega t) \text { at } \mathrm{y}=0
$$

where $v$ is the imposed boundary condition, $\omega$ is a prescribed frequency for oscillatory actuation, $A_{0}$ is an amplitude and $\mathrm{f}_{0}(\mathrm{x})$ is a spatial distribution, which together represent a steady momentum suction or blowing coefficient, and $A_{1}$ is an amplitude and $f_{1}(x)$ is a spatial distribution, which together represent an oscillatory mass coefficient. Rather than synthetic-jet like actuation, a slight deviation of the temporal forcing could produce pulsed vortex generator jets (PVGJ). ${ }^{9}$

To move from research to application, tools are required which can predict the momentum information $\left(A_{0}, f_{0}\right)$ and/or $\left(A_{1}, f_{1}\right)$ required for active flow control CFD. While the steady suction or blowing components $\left(A_{0}, f_{0}\right)$ can readily be obtained from conservation of mass flow from the actuator, the zero-net-mass actuation prediction must involve the modeling of the whole actuator. In the next section, actuator modeling tools will be discussed and compared with experimental observation for a synthetic jet actuator.
Only structural-fluid modeling related to synthetic jet actuators will be considered in this section. The basic components of a synthetic jet device are a cavity and oscillating material(s). A piezoelectric material drives the oscillating material, which typically is a flexible diaphragm or a thin metal plate..$^{22}$ Better performance occurs if several piezoelectric actuators are strategically placed within each cavity. The main function of the stand-alone synthetic jet device is to drive air through a hole or slot in order to generate a train of vortices that are formed at the edge of the hole or slot.

\subsection{Synthetic Jet Modeling}

Various synthetic jet concepts have been studied using diaphragms with piezoelectric material for actuation. To properly size and to evaluate their effectiveness one must be able to predict the performance of such devices given a certain geometry and actuator configuration. A design used in this particular study uses a dual circular diaphragm with piezoelectric material bonded onto each diaphragm. Both diaphragms are clamped or hinged onto the sides of a cubical enclosure with a narrow slot to allow air in and out of the enclosure (Figure 6).

A simple assessment of the pump performance can be made by assuming that incompressible fluid is inside the enclosure cavity. In this case, jet velocity is a function of volumetric changes caused by diaphragm motion. Compressibility effects may be very important if a prediction of the actual flow speed exiting the slot is the final goal. In this initial study, our goal is to develop a simple mathematical tool to determine an upper bound on the maximum achievable jet velocity given a certain geometry and actuator.

\section{ACTUATOR MODELING}



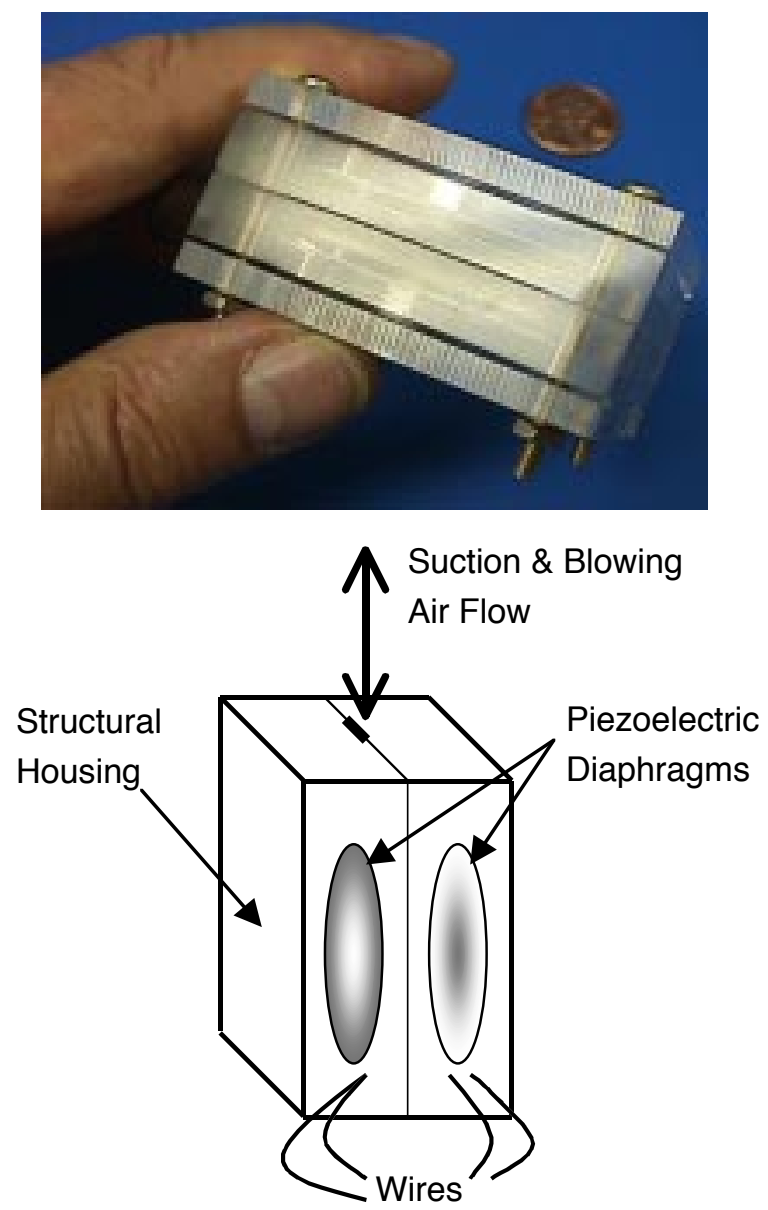

Figure 6. Picture and sketch of dual-diaphragm synthetic jet actuator.

Considering a circular diaphragm, the solution to the differential equation describing the diaphragm motion was given in Ref. 23 as

$$
\begin{aligned}
& w(r, t)=\sum_{n=1}^{\infty} z_{n}(r, \theta) \cos \left(\omega_{n} t\right) \\
& z_{n}(r, \theta)=\left[A_{n} J_{n}(k r)+C_{n} I_{n}(k r)\right] \cos (n \theta)
\end{aligned}
$$

where $\omega_{n}$ is the $\mathrm{n}^{\text {th }}$ natural frequency, and $w(r, t)$ is the diaphragm out-of-plane displacement written in terms of products of a time function and $z_{n}(r, \theta)$. The component $z_{n}(r, \theta)$ captures the radial and circular variation of the diaphragm displacement. Radial components are given in terms of Bessel functions $J_{n}(\mathrm{kr})$ and modified Bessel functions of the first kind, $I_{n}(k r)$. The index $\mathrm{k}$ is computed using the boundary conditions. For a clamped circular plate with radius $r=a$, the boundary condition along the constrained edge is $z_{n}(a, \theta)=\partial z_{n}(r, \theta) /\left.\partial r\right|_{r=a}=0$. In the pinned (simply supported) case, the boundary condition is $z_{n}(a, \theta)=M_{r}(a)=0$ where $M_{r}(a)$ is the radial component of the moment along at the boundary. Solutions for various boundary conditions are also documented $^{23}$ as a function of the parameter $\lambda=\mathrm{k} a$. For clamped boundary condition, the first $\lambda^{2}$ solution is 10.216 . Similarly for the pinned condition, the first $\lambda^{2}$ solution is 4.977 . These solutions are related to the natural frequency of the plate by $\lambda^{2}=\omega a^{2} \sqrt{\rho h / D}$, where $\omega$ is the natural frequency of the plate, $\rho$ is the material density, $\mathrm{h}$ is the plate thickness, and $D=E h^{3} / 12\left(1-v^{2}\right)$ is the plate rigidity. The plate rigidity is computed using Young's Modulus E for the material, Poisson's ratio $v$, and the plate thickness $\mathrm{h}$.

To evaluate the performance of a synthetic jet actuator, consider a diaphragm vibrating at its first natural frequency, as shown in Figure 7.

Volumetric changes due to diaphragm motion can be easily estimated when the system is vibrating at resonance. The volume changes generated by the diaphragm motion are computed by considering the area under the curve in Figure 8 (cross-section in the w-r plane) and Pappus' Theorem for a solid of revolution. ${ }^{24}$ The volume generated by rotating the area under the curve in Figure 8 about the $w$ axis in the $w-r$ plane is $V=2 \pi a^{2} Y_{n} \bar{A}_{n} \bar{r}_{n}$, where $\bar{A}_{n}$ is the normalized area for mode $\mathrm{n}, \bar{r}_{n}$ is the corresponding area centroid, and $Y_{n}$ is the maximum displacement for mode $\mathrm{n}$. Normalization is with respect to the maximum displacement and are defined by 


$$
\begin{gathered}
\bar{A}_{n}=\frac{1}{Y_{n}} \int_{0}^{1} z_{n}(\eta a, 0) d \eta, \\
\bar{r}_{n}=\frac{1}{Y_{n} \bar{A}_{n}} \int_{0}^{1} z_{n}(\eta a, 0) \eta d \eta, \\
Y_{n}=\max \left(z_{n}(r, \theta)\right)
\end{gathered}
$$

The volumetric rate of change is written as

$$
\frac{d V}{d t}=2 \pi a^{2} \sum_{n=1}^{\infty} Y_{n} \bar{A}_{n} \bar{r}_{n} \omega_{n} \sin \left(\omega_{n} t\right)
$$

For a dual diaphragm configuration this rate of change must be multiplied by a factor of 2 .

Estimated jet velocity is now given by

$$
\begin{aligned}
v & =\frac{2}{C_{d} A_{s}} \frac{d V}{d t} \\
& =\frac{2}{C_{d} A_{s}} 2 \pi a^{2} \sum_{n=1}^{\infty} Y_{n} \bar{A}_{n} \bar{r}_{n} \omega_{n} \sin \left(\omega_{n} t\right) \\
v & =\frac{4}{C_{d}}\left(\frac{A_{d}}{A_{s}}\right) \sum_{n=1}^{\infty} Y_{n} \bar{A}_{n} \bar{r}_{n} \omega_{n} \sin \left(\omega_{n} t\right)
\end{aligned}
$$

where $A_{s}$ is the area of the slot, $A_{d}$ is the area of the diaphragm, and $C_{d}$ is a discharge coefficient to account for energy losses in the exit slot. In designing a synthetic jet, the first mode of the diaphragm dominates the system response.

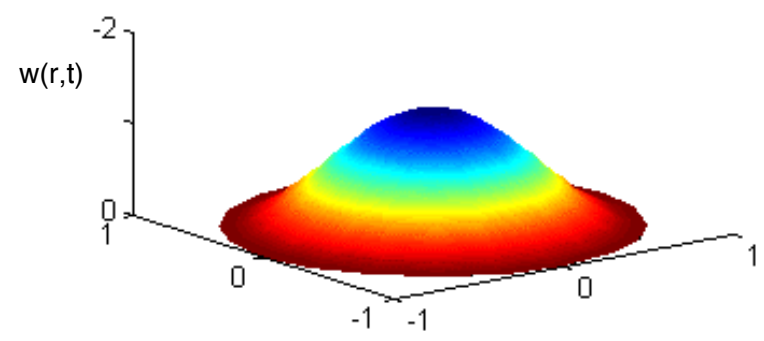

Figure 7. First bending mode of circular diaphragm.

A non-dimensional relation among the parameters defined in equation (5) can be written for the first mode

$$
\frac{v}{Y_{1} \omega_{1}}=\frac{4 \bar{A}_{1} \bar{r}_{1}}{C_{d}} \frac{A_{d}}{A_{s}}
$$

where $v / Y_{1} \omega_{1}$ is the maximum velocity ratio and $A_{s} / A_{d}$ is an area ratio. Parameters for clamped and pinned boundary conditions are given in Table 1.

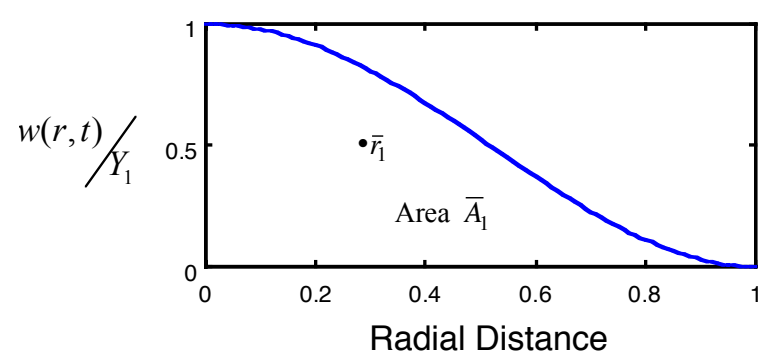

Figure 8. Diaphragm displacement using Bessel function for mode 1.

Table 1. Normalized area and centroid for first mode.

\begin{tabular}{|l|c|c|}
\hline \multicolumn{1}{|c|}{$\begin{array}{c}\text { Boundary } \\
\text { Condition }\end{array}$} & $\bar{A}_{1}$ & $\bar{r}_{1}$ \\
\hline Clamped & 0.5132 & 0.3036 \\
\hline Pinned & 0.6251 & 0.3589 \\
\hline
\end{tabular}

Figure 9 shows a design curve for the boundary conditions in Table 1 and a unit discharge coefficient $C_{d}=1$. Both boundary conditions are shown because experimentally it is difficult to ensure that a "true" pinned condition is achieved. Design parameters are in terms of diaphragm area, slot area, fundamental frequency of diaphragm, and the maximum diaphragm displacement.

The analysis thus far does not account for piezoelectric actuator effects explicitly. The actuator effectiveness is a function of material used, actuator bonding approach, and boundary conditions. Modeling of these effects although possible is not included in this analysis at this time. Instead, experimental data from stand-alone actuator test is used to demonstrate actuator 
effectiveness in terms of maximum diaphragm displacement. For example, a diaphragm with a radius $a=25 \mathrm{~mm}$ made of brass with a thickness of $\mathrm{h}=0.20 \mathrm{~mm}$ has a frequency response as shown in Figure 10 when clamped. The frequency response is computed between mid-diaphragm displacement to actuator input voltage. Examining the low frequency range one can determine that the diaphragm moves about $0.05 \mathrm{~mm}$ with a 100 volts input whereas at resonance the displacement is up to $0.53 \mathrm{~mm}$. In this configuration, the clamped natural frequency is $849 \mathrm{~Hz}$. For the pinned condition, the natural frequency is $346 \mathrm{~Hz}$. It is important to note that the maximum diaphragm displacement at resonance is inversely proportional to the damping level and varies significantly with bonding method and boundary conditions. Data for various actuator configurations needs to be developed.

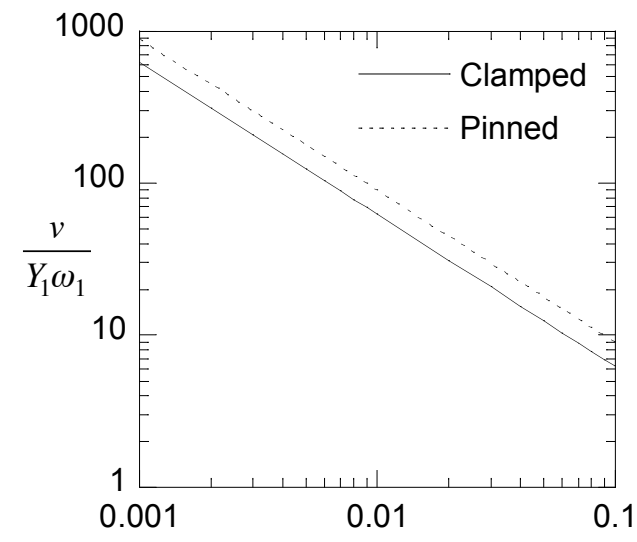

$\operatorname{Area} \operatorname{Ratio}\left(A_{s} / A_{d}\right)$

Figure 9. Dual diaphragm design curve for $C_{d}=1$.

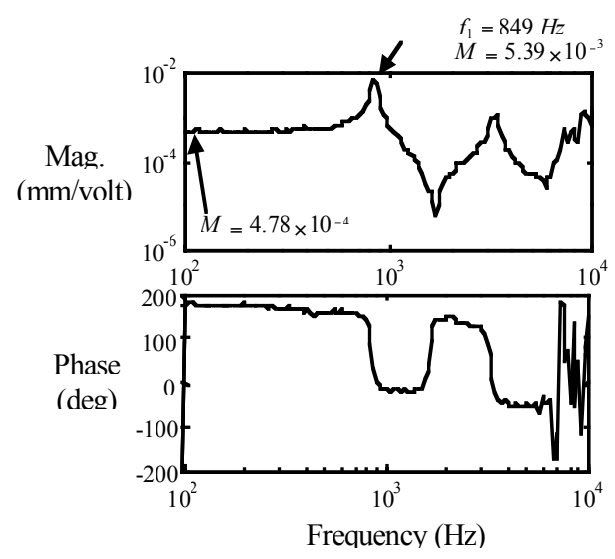

Figure 10. Frequency response of clamped brass diaphragm.

\subsection{Validation of Synthetic Jet Model}

To validate the actuator model, a synthetic jet device was built and tested on a laboratory bench top. ${ }^{22} \mathrm{~A}$ small housing in which a cylindrical cavity was enclosed by two brass diaphragms $(50.0 \mathrm{~mm}$ in diameter and $0.20 \mathrm{~mm}$ in thickness) were placed opposite each other. A piezoelectric material with a diameter of $23 \mathrm{~mm}$ was attached to the center of outside face of each brass diaphragm. The pair of piezoelectric brass diaphragms were operated with a $180^{\circ}$ phase differential at the same voltage and frequency.

With actuation, a synthetic jet issued from the 35.5 $\mathrm{mm}$ long by $0.50 \mathrm{~mm}$ wide slot on the top of the device (Figure 6). A streak photograph of the synthetic jet operating in still air environment is shown in Figure 11. This picture is a normal plane view across the middle of the slot length. One component hot-wire measurements were made at the actuator slot exit. At the resonant frequency of $350 \mathrm{~Hz}$, a maximum velocity of $40 \mathrm{~m} / \mathrm{s}$ was measured. At this frequency, the measured maximum displacement at the center of diaphragm is $0.24 \mathrm{~mm}$. Using the above non-dimensional relationships, the current actuator leads to $A_{s} / A_{d}=0.01$ and $v / Y_{1} \omega_{1}=70$, which is very close to the pinned-condition line of Figure 9. 


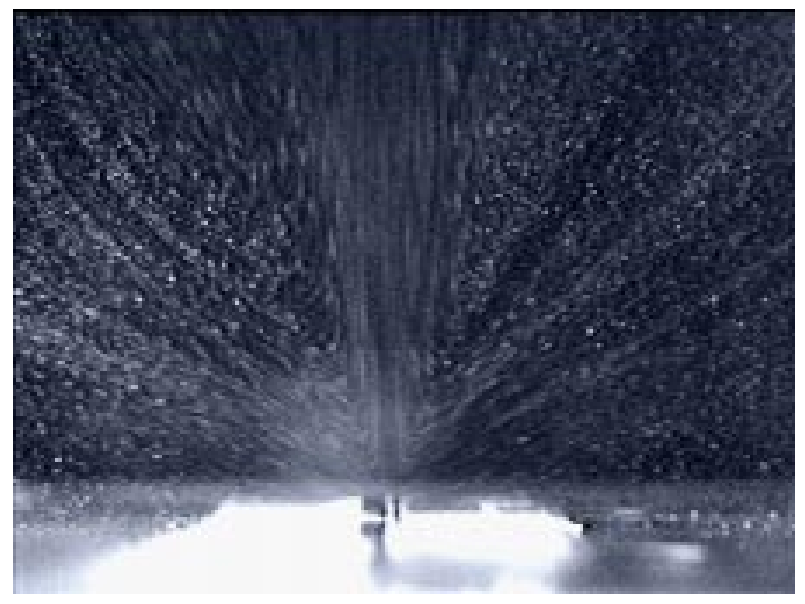

Figure 11. Streak photograph of actuator induced flow.

\section{CONCLUDING REMARKS}

This presentation has focused on a strategy to develop active flow control design tools to enable the transition of active flow control from the laboratory to applications. Clearly, significant amount of additional research is required before a production-type tool is available for the design engineer; however, progress is being made at NASA, at various universities, and in the aircraft industry toward developing these necessary tools. Also, numerous additional flow control experiments are underway to provide necessary databases for validation. A number of advancements have been made within the industry IRAD projects, which we regrettable could not mention here for proprietary reasons.

\section{ACKNOWLEDGMENTS}

The author is indebted to Dr. Avi Seifert, Tel-Aviv University, for supplying the geometry and experimental data for the TAU0015 model. Also, thanks to Dr. W. Kyle Anderson, NASA Langley Research Center, for use of the FUN2D code. This work was supported the NASA Airframe Systems Program Office, Aircraft Morphing program.

\section{REFERENCES}

${ }^{1}$ Gad-el-Hak, M., Flow Control. Applied Mechanics Review, Vol. 42, No. 10, 1989, pp. 261-293.

${ }^{2}$ Gad-el-Hak, M., and Bushnell, D. M., Separation Control: Review. J. Fluids Engineering, Vol. 113, 1991, pp. 5-30.

${ }^{3}$ Chang, P. K. Control of Flow Separation: Energy Conservation, Operational Efficiency, and Safety. Hemisphere Publishing Corp., Washington DC, 1976.

${ }^{4}$ Lin, J. C. Control of Turbulent Boundary-Layer Separation using Micro-Vortex Generators. 30th AIAA Fluid Dynamics Conference, Norfolk, VA/June 28-July 1, 1999. (AIAA Paper 99-3404)

5Joslin, R. D. Overview of Laminar Flow Control. NASA Technical Paper 208705, October 1998.

${ }^{6}$ McLean, J. D., Crouch, J. D., Stoner, R. C., Sakurai, S., Seidel, G. E., Feifel, W. M., and Rush, H. M. Study of the Application of Separation Control by Unsteady Excitation to Civil Transport Aircraft. NASA/CR-1999-209338, June 1999.

${ }^{7}$ Chandrasekhara, M. S., Wilder, M. C., Carr, L. W. Control of Flow Separation Using Adaptive Airfoils. $35^{\text {th }}$ Aerospace Sciences Meeting \& Exhibit, Reno, NV/January 6-10, 1997. (AIAA Paper 97-0655)

${ }^{8}$ Seifert, A., Eliahu, S., Greenblatt, D. and Wygnanski, I. Use of Piezoelectric Actuators for Airfoil Separation Control. AIAA Journal, Vol. 36, No. 8, 1998, pp. 1535-1537.

${ }^{9}$ McManus, K. and Magill, J. Separation Control in Incompressible and Compressible Flows using Pulsed Jets. $27^{\text {th }}$ AIAA Fluid Dynamics Conference, New Orleans, LA/June 17-20, 1996. (AIAA Paper 96-1948) 
${ }^{10}$ Seifert, A., Bachar, T., Wygnanski, I., Kariv, A., Cohen, $\mathrm{H}$. and Yoeli, R. Application of Active Separation Control to a Small Unmanned Air Vehicle. Journal of Aircraft, Vol. 36, No. 2, 1998, pp. 474-477.

${ }^{11}$ Seifert, A. and Pack, L. G. Oscillatory Excitation of Unsteady Compressible Flows over Airfoils at Flight Reynolds Numbers. $37^{\text {th }}$ AIAA Aerospace Sciences Meeting \& Exhibit, Reno, NV/January 11-14, 1999. (AIAA Paper 99-0925)

${ }^{12}$ Pack, L. G. and Seifert, A. Periodic Excitation for Jet Vectoring and Enhanced Spreading. $37^{\text {th }}$ AIAA Aerospace Sciences Meeting \& Exhibit, Reno, NV/January 11-14, 1999. (AIAA Paper 99-0672)

${ }^{13}$ Kral, L. D., Donovan, J. F., Cain, A. B., and Cary, A. W., Numerical Simulation of Synthetic Jet Actuators. 28th AIAA Fluid Dynamics Conference, Snowmass Village, CO/June 29-July 2, 1997.

(AIAA Paper 97-1824)

${ }^{14}$ Joslin, R. D., Gunzburger, M. D., Nicolaides, R. A., Erlebacher, G., and Hussaini, M. Y., A SelfContained, Automated Methodology for Optimal Flow Control-Application to Transition Delay. AIAA Journal, Vol. 35, No. 5, May 1997, pp. 816824.

${ }^{15}$ Ravindran, S. S., Proper Orthogonal Decomposition in Optimal Control of Fluids. NASA/TM-1999-209113, March 1999.

${ }^{16}$ Anderson, W. K. and Bonhaus, D. L., An Implicit Upwind Algorithm for Computing Turbulent Flows on Unstructured Grids. Computers Fluids, Vol. 23, No. 1, 1994, pp. 1-21.
${ }^{17}$ Spalart, P. R., and Allmaras, S. R., A OneEquation Turbulence Model for Aerodynamic Flows. 30th Aerospace Sciences Meeting \& Exhibit, Reno, NV/January 6-9, 1992. (AIAA Paper 92-0439).

${ }^{18}$ Donovan, J. F., Kral, J. D., and Cary, A. W., Active Flow Control Applied to an Airfoil. 36th AIAA Aerospace Sciences Meeting \& Exhibit, Reno, NV/January 12-15, 1998.

(AIAA Paper 98-0210).

${ }^{19}$ Williamson, C. H. K., Vortex Dynamics in the Cylinder Wake. Annual Review of Fluid Mechanics, Vol. 28, 1996, pp. 477-539.

${ }^{20}$ Franke, R., Rodi, W., and Schl"onung, B., Numerical Calculation of Laminar Vortex-Shedding Flow Past Cylinders. Journal of Wind Engineering and Industrial Aerodynamics, Vol. 35, 1990, pp. 237-257.

${ }^{21}$ Smith, B. L. and Glezer, A. The Formation and Evolution of Synthetic Jets. Physics of Fluids, Vol. 10, No. 9, September 1998, pp. 2281-2297.

${ }^{22}$ Bryant, R. G., Fox, R. L., Lachowicz, J. T., and Chen, F.-J. Piezoelectric Synthetic Jets for Aircraft Control Surfaces. Smart Structures and Materials $6^{\text {th }}$ Annual International Symposium, Newport Beach, CA/March 1-5, 1999.

${ }^{23}$ Leissa, A. W., Vibration of Plates. NASA SP-160, 1969.

${ }^{24}$ Salas, S. L. and Hide, E., Calculus: One and Several Variables. $2^{\text {nd }}$ Ed., 1971. 